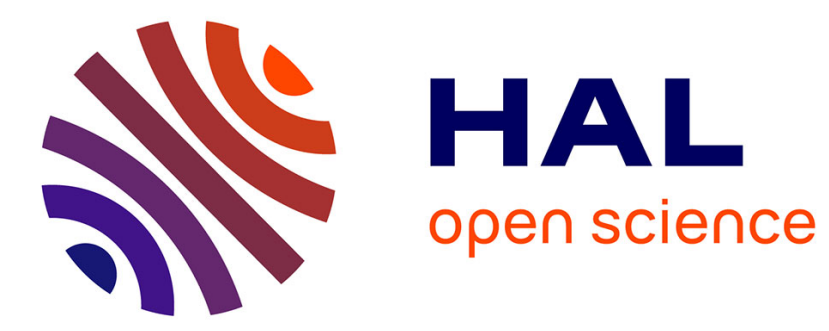

\title{
First principles calculation of noble gas atoms properties in $3 \mathrm{C}-\mathrm{SiC}$
}

Laurent Pizzagalli

\section{To cite this version:}

Laurent Pizzagalli. First principles calculation of noble gas atoms properties in 3C-SiC. Journal of Nuclear Materials, 2012, 429 (1-3), pp.329. 10.1016/j.jnucmat.2012.06.022 . hal-00728924

\section{HAL Id: hal-00728924 \\ https://hal.science/hal-00728924}

Submitted on 7 Sep 2012

HAL is a multi-disciplinary open access archive for the deposit and dissemination of scientific research documents, whether they are published or not. The documents may come from teaching and research institutions in France or abroad, or from public or private research centers.
L'archive ouverte pluridisciplinaire HAL, est destinée au dépôt et à la diffusion de documents scientifiques de niveau recherche, publiés ou non, émanant des établissements d'enseignement et de recherche français ou étrangers, des laboratoires publics ou privés. 


\title{
First principles calculation of noble gas atoms properties in 3C-SiC
}

\author{
A. Charaf Eddin and L. Pizzagalli, \\ Departement of Physics and Mechanics of Materials, Institut P', CNRS-Université de Poitiers-ENSMA UPR 3346, SP2MI, BP 30179, \\ F-86962 Futuroscope Chasseneuil Cedex, France
}

\begin{abstract}
First-principles calculations were performed to investigate the properties of single noble gas atoms (He, Ne, Ar, $\mathrm{Kr}$, Xe) in 3C-SiC. Three cases were considered: (i) a noble gas atom in a perfect crystal, (ii) in the neighborhood of a monovacancy, (iii) and of a divacancy. For each case the stable configurations were determined, as well as their formation energies. The mobility of He, Ne and $\mathrm{Ar}$ interstitials was studied, and the associated migration energies were calculated. Our results were discussed and compared to available experimental or theoretical works.
\end{abstract}

Key words: Noble gas; Silicon Carbide; Density functional theory; Diffusion PACS: 61.72.U-, 81.05.Je, 31.15.E, 66.30.J-

\section{Introduction}

Silicon carbide is a material exhibiting several outstanding properties, in particular in extreme conditions like high temperatures or high mechanical stresses [1]. As such, it is considered a promising candidate for applications in harsh environment. In nuclear context, $\mathrm{SiC}$ is potentially interesting as a structural material in future fusion reactors and as a fuel cladding material in next generation fission reactors. In both situations, the material can be exposed to a significant production of helium. Other heavier noble gas (NG) such as Kr or Xe can also be introduced from nuclear transmutation and decay. All NG species have a very low solubility, and are known to precipitate and form extended defects such as bubbles or platelets in many systems [2]. Those defects can be sources of several mechanisms such as swelling, surface blistering, plane cleavage, which will irreversibly degrade the material properties. Finally, He in $\mathrm{SiC}$ is also an interesting system in a spatial context, since $\mathrm{He}$ (and $\mathrm{Ne}$ ) can be found in SiC dust grains present in primitive meteorites [3].

Although there have been many studies dedicated on NG atoms behavior in metals [4] or in silicon [5-7], the literature is much more scarce for $\mathrm{SiC}$. The pioneering works of Hojou and co-workers [8,9] and of Jung and co- workers [10-12] revealed the formation of He-filled bubbles and platelets in $\mathrm{SiC}$ depending on the conditions. The effect of the microstructure on these bubbles was also studied [13]. Other NG species such as Ne and Xe were shown to also lead to bubbles formation [14]. To understand this bubble formation process, it is first necessary to gain information on several properties related to a single NG atom. For instance, the location of the interstitial NG atom in the lattice, its formation and migration energies, the interaction of this impurity with vacancies, are all essential quantities that must be known before building larger scale models of bubble formation and evolution. For these quantities, almost all what is known is concerning helium. Experimentally, although the exact location of a He interstitial is difficult to estimate [15], its migration energy was measured to be $1.5 \mathrm{eV}$ at $600 \mathrm{~K}$ [16] and $1.14 \mathrm{eV}$ for temperatures ranging from $800 \mathrm{~K}$ to $1050 \mathrm{~K}$ [17]. The detrapping of He from bubbles or small He-vacancies complexes was also investigated and activation energies ranging from $3.2 \mathrm{eV}$ to $4.0 \mathrm{eV}$ were proposed $[16,18]$. There are also a handful of dedicated numerical simulations [19-22], but yielding contradictory results. In fact, although all studies agree that the most stable location for a He interstitial is the tetrahedral site surrounded by four Si atoms, a wide range of formation energies, from $1.51 \mathrm{eV}$ [19] to $3.47 \mathrm{eV}[22]$, is proposed. Finally, only one work focused 


\begin{tabular}{cccc}
\hline $\mathrm{Si}_{T S i}$ & $\mathrm{Si}_{T C}$ & $\mathrm{SiSi}_{<100>}$ & $\mathrm{SiSi}_{<110>}$ \\
\hline $11.69(9.40)$ & $10.16(7.25)$ & $10.65\left(\rightarrow \mathrm{Si}_{T C}\right)$ & $9.16(8.12)$ \\
\hline $\mathrm{C}_{T S i}$ & $\mathrm{C}_{T C}$ & $\mathrm{CC}_{<100>}$ & $\mathrm{CC}_{<110>}$ \\
\hline $9.78(10.00)$ & $11.33\left(6.72^{*}\right)$ & $6.86(6.31)$ & $7.15(6.63)$ \\
\hline
\end{tabular}

Table 1

Formation energies (in eV) of different 3C-SiC self interstitials in 64 and 216 (in parentheses) atoms cells. (*) In the 216 atoms cell, $\mathrm{C}_{T C}$ is not stable and evolves to a $\mathrm{CC}_{<111>}$ configuration.

on the interaction of He with vacancies and the calculation of interstitial migration energy [19]. Regarding the other NG species in SiC, Bertolus investigated the stability of $\mathrm{Kr}$ and Xe in presence of mono and divacancies [23].

We report in this paper a comprehensive study of the properties of a single NG atom ( $\mathrm{He}, \mathrm{Ne}, \mathrm{Ar}, \mathrm{Kr}$ and $\mathrm{Xe}$ ) in $3 \mathrm{C}-\mathrm{SiC}$, from density functional theory calculations. In particular, we determined the most stable location for an interstitial configuration, and also in interaction with mono and divacancies. Finally, we also determined the migration energy of the interstitial for light NG species.

\section{Methods}

Our calculations were done in the framework of Density Functional Theory (DFT) [24,25], using the plane wave self consistent field PWscf code [26]. The interaction of valence electrons with the ions was described with ultrasoft pseudopotentials [27]. We used periodic boundary conditions for modelling an infinite crystal, and cubic systems including 64 or 216 atoms. The Brillouin zone was sampled with a $3 \times 3 \times 3$ grid of $k$-points [28] for the smallest system, whereas a single $k$ point $\Gamma$ was found sufficient for the latter. The Perdew-Burke-Ernzerhof Generalized-Gradient Approximation (PBE-GGA) [29] was used for describing exchange and correlation, since it allows an overall better description of weak bonding than Local Density Approximation (LDA) [25]. We found that the electronic structure was accurately converged with a plane-wave cutoff energy of 30 Ry. Using these parameters, we have determined the lattice parameter $a_{0}$ of $3 \mathrm{C}-\mathrm{SiC}$ to be equal to $4.376 \AA$, close to the experimental value of $4.359 \AA$.

To assess the validity of our computational setup, different types of $\mathrm{SiC}$ self interstitials, tetrahedral $\mathrm{T}$, split $<100>$ and $<110>$, have been first modelled, the calculated formation energies being shown in Table 1. For Si interstitials, the most stable configurations are obtained for a carbon tetrahedral $\mathrm{T}$ and $<110>$ split interstitial configurations, in agreement with the literature [30-33]. For the $\mathrm{C}$ interstitial, we found the lowest formation energies for $<100>$ and $<110>$ CC split interstitials, also in agreement with previous works $[30,31,34]$. The formation energies decrease (for most defects) as the supercell gets larger, due to the increase of the defect relaxation volume.
Finally, in order to compute the NG atom migration in $\mathrm{SiC}$, the Nudged Elastic Band technique (NEB) $[35,36]$ has been used with three intermediate optimized images, and with the supercell of 64 atoms.

\section{NG interstitials}

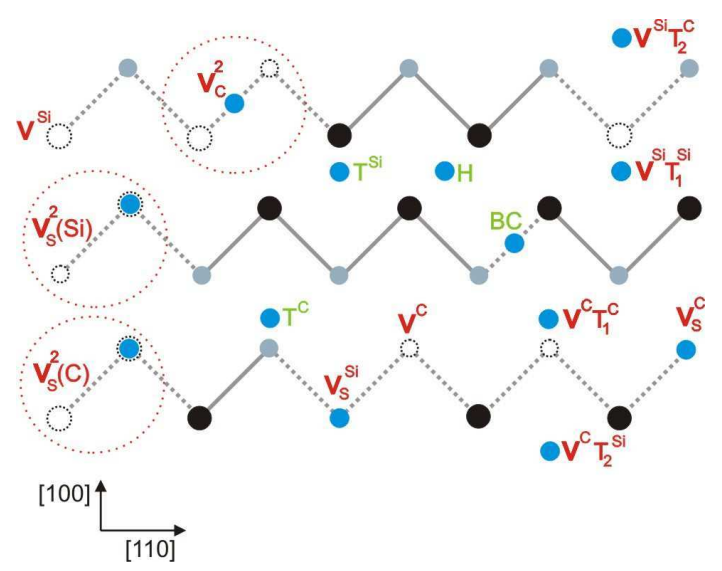

Fig. 1. High symmetry NG configurations in 3C-SiC shown in the plane of the [110] zigzag chains. Si (C) atoms are shown as black (grey) circles. Si and $\mathrm{C}$ vacancies are represented by empty dashed line circles, whereas blue circles are used for NG atoms (color for electronic version).

Our first goal is to find the most stable configuration for a single NG atom in 3C-SiC bulk. We initially supposed that NG atoms prefer to be located as far away as possible from $\mathrm{Si}$ and $\mathrm{C}$ atoms, as in $\mathrm{Si}$ [37-39]. Thus, two high symmetry possible candidates are the tetrahedral (T) and hexagonal (H) interstitial configurations (Fig. 1). Since $3 \mathrm{C}-\mathrm{SiC}$ is composed of two different species, there are two possible T sites: $\mathrm{T}^{S i}$ is the case where the four 1stneighbors of the NG atom are $\mathrm{Si}$ atoms, and $\mathrm{T}^{C}$ where the four 1st-neighbors of the $\mathrm{NG}$ atom are $\mathrm{C}$ atoms. On the other hand, there is a single $\mathrm{H}$ configuration, the six 1stneighbors being three $\mathrm{Si}$ atoms and three $\mathrm{C}$ atoms, forming a stoichiometric ring around the NG atom (Fig. 1).

The formation energies $E_{f}$, reported in Table 2 for these configurations, are computed according to

$E_{f}=E_{S i C+N G}-n\left(\frac{\mu_{S i C}}{2}\right)-\mu_{N G}$

$\mu_{S i C}$ is the chemical potential of a pair of one silicon and one carbon atoms in $\mathrm{SiC}$, calculated using a bulk of $n=64$ (or 216) atoms, whereas $\mu_{N G}$ is the energy of the single NG atom in the empty reference cell. 
$(a)$

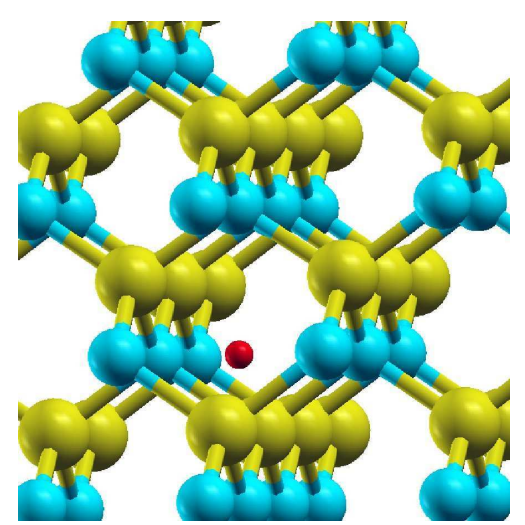

(b)

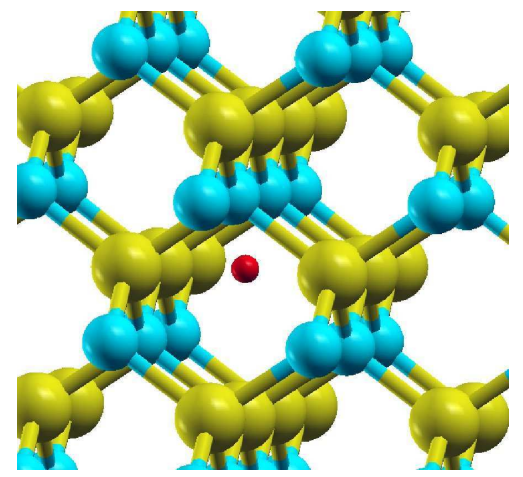

Fig. 2. He $\mathrm{T}^{S i}(a)$ and $\mathrm{T}^{C}(b)$ interstitial configurations after relaxation. Si atoms are shown with yellow spheres, whereas blue spheres are used for $\mathrm{C}$ atoms. The size of the sphere representing the $\mathrm{He}$ atom (in red) is proportional to its (estimated) core radius (color for electronic version).

Our results indicate that the $\mathrm{T}^{S i}$ and $\mathrm{T}^{C}$ configurations are stable for all NG atoms, i.e. the NG atom stays in its initial position after structural relaxation, as shown in Fig. 2 for the He atom. However it is not the case for the $\mathrm{H}$ site. The latter is found to be stable only for $\mathrm{Kr}$ and Xe atoms, with a significant lattice distortion. All other atoms, i.e. $\mathrm{He}, \mathrm{Ne}$ and $\mathrm{Ar}$, located in a $\mathrm{H}$ site were unstable, and moved during relaxation towards the nearest $\mathrm{T}^{\mathrm{Si}}$ configuration. The unstability could be simply explained by the presence of two types of atoms in the zinc-blende crystal, which leads to two different $\mathrm{T}$ configurations, $\mathrm{T}^{\mathrm{Si}}$ and $\mathrm{T}^{C}$. A NG atom initially located in a $\mathrm{H}$ site could relax into one of those two sites. Since two different energy gains are possible when He or Ne relax in $\mathrm{T}^{S i}$ or $\mathrm{T}^{C}$, the energy curvature in $\mathrm{H}$ is asymmetric. The weak stability observed in bulk silicon [37] is lost and the relaxation in $\mathrm{T}^{S i}$ is favored. However, for large NG atoms such as $\mathrm{Kr}$ and $\mathrm{Xe}$, the deformation is large enough to trap the NG atom in the lattice, like for Xe in silicon [37].

We checked another possible configuration, the bond centered interstitial (BC) shown in Fig. 1, characterized by the NG atom located between first neighbors Si and C atoms. Our results indicate that this configuration is stable for all species. During structural relaxation, the Si and C close to the NG atom move away from each other, forming

\begin{tabular}{ccccc}
\hline $\mathrm{NG}$ & $\mathrm{T}^{S i}$ & $\mathrm{~T}^{C}$ & $\mathrm{H}$ & $\mathrm{BC}$ \\
\hline $\mathrm{He}$ & $3.07(3.04)$ & $3.54(3.51)$ & $\rightarrow \mathrm{T}^{S i}$ & $7.42(7.03)$ \\
\hline $\mathrm{Ne}$ & $7.51(7.45)$ & $8.58(8.49)$ & $\rightarrow \mathrm{T}^{S i}$ & $10.59(9.95)$ \\
\hline $\mathrm{Ar}$ & $15.58(15.40)$ & $17.25(16.86)$ & $\rightarrow \mathrm{T}^{S i}$ & $14.61(13.30)$ \\
\hline $\mathrm{Kr}$ & $20.07(19.77)$ & $21.26(20.57)$ & 19.86 & $16.75(15.30)$ \\
\hline $\mathrm{Xe}$ & $26.71(24.00)$ & $26.33(23.21)$ & 25.00 & $20.14(18.49)$ \\
\hline
\end{tabular}

Table 2

Formation energies (in eV) of possible NG interstitial configurations in 64 and 216 (in parentheses) atoms cells.

a three-centers aligned Si-NG-C, accompanied by a lattice deformation which increases as the NG species gets larger. The distance between $\mathrm{Si}$ and $\mathrm{C}$ increases by $70 \%$ for $\mathrm{He}$, and by $128 \%$ for Xe.

All interstitials formation energies are reported in Table 2 . In the case of $\mathrm{He}$ and $\mathrm{Ne}$, the most stable configuration is $\mathrm{T}^{S i}$. For heaviest NG atoms, such as $\mathrm{Ar}, \mathrm{Kr}$ and $\mathrm{Xe}$, we found that the $\mathrm{BC}$ configuration has the lowest energy of all structures. For all species, the $\mathrm{H}$ and $\mathrm{T}^{C}$ configurations are never the most stable. It is clear from Table 2 that results using 64 or 216 atoms supercell are qualitatively in agreement, but more accurate formation energies are expected in the case of the 216 atoms supercell, especially for large NG atoms like $\mathrm{Kr}$ and Xe.

Both $\mathrm{T}^{S i}$ and $\mathrm{T}^{C}$ configurations were considered for He in previous studies using a cell of 64 atoms, by Van Ginhoven et al [19] (1.51 and $2.71 \mathrm{eV}$ respectively) and Kim et al [20] (2.87 and $3.31 \mathrm{eV}$ respectively). There is a large difference between those results, especially for the $\mathrm{T}^{S i}$ configuration. Our own calculations yield values in rather close agreement with formation energies from Kim et al [20]. Van Ginhoven et al used different computational parameters, with the $\Gamma$ point only and the local density approximation. In comparison, Kim et al performed more accurate calculations with a finer $4 \times 4 \times 4$ grid of $k$ points and the generalized gradient approximation, pretty much the same as in our work. As a test, we have computed formation energies using $\Gamma$ point only. We found $2.97 \mathrm{eV}$ and $3.45 \mathrm{eV}$ for $\mathrm{T}^{S i}$ and $\mathrm{T}^{C}$ respectively, then close to our well converged calculations and still in disagreement with values from Van Ginhoven et al [19]. It is possible, although unlikely, that the disagreement could be explained by the use of LDA in their work, and GGA-PBE in ours. Nevetherless, all studies agree on the fact that the $\mathrm{T}^{S i}$ configuration is most stable than the $\mathrm{T}^{C}$ one. A possible explanation has been proposed by Van Ginhoven et al [19]. They suggested that the relative electronegativity of carbon versus silicon would favor excess electron density on the $\mathrm{C}$ atom, thus increasing the steric repulsion in the $\mathrm{T}^{C}$ geometry (since the NG atom is surrounded by $4 \mathrm{C}$ ). It is also likely that changes in the electronic structure due to displacement of the 1st-neighbor atoms, would be energet- 
ically cheaper for $\mathrm{Si}$ than for $\mathrm{C}$ atoms. Therefore, a small NG atom like He or Ne tends to be located in $\mathrm{T}^{S i}$ where interactions are weaker, and less deformations are needed.

For Ar, Kr and Xe species, the BC configuration is the most stable one as shown in Table 2. In such a configuration it is necessary to break a bond between the $\mathrm{C}$ and the Si atoms, which are largely displaced from their ideal positions. Nevertheless, the deformation for the other lattice atoms is overall reduced compared to tetrahedral configurations. For the bulky NG atoms, the elastic energy gain is large enough to explain why the $\mathrm{BC}$ configuration is more stable. This is confirmed by the fact that the formation energy difference between $\mathrm{BC}$ and other configurations increases as the NG atom gets larger. Bertolus recently performed interstitial calculations for $\mathrm{Kr}$ and $\mathrm{Xe}$ [23], and found formation energies in very close agreement to ours.

\section{NG monovacancy complex}

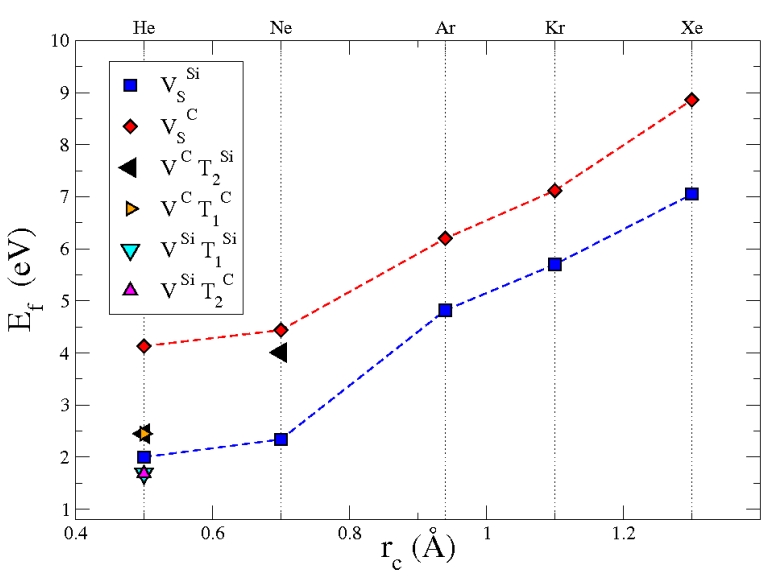

Fig. 3. Formation energies (in eV) of NG monovacancy complexes calculated using a $64 \mathrm{Si}$ atoms cell for different $\mathrm{NG}$ species as a function of the (estimated) core radius $\mathrm{r}_{C}$ (in $\AA$ ) (color for electronic version).

Removing one atom of the $3 \mathrm{C}-\mathrm{SiC}$ crystal leads to the creation of a monovacancy. Due to the binary nature of $\mathrm{SiC}$, two types of monovacancy are possible: $\mathrm{V}^{S i}$ or $\mathrm{V}^{C}$. These configurations were modeled and their formation energies calculated using the same expression as before. For a supercell of 64 atoms, we obtained $7.40 \mathrm{eV}$ for $\mathrm{V}^{S i}$ and $4.67 \mathrm{eV}$ for $\mathrm{V}^{C}(7.71 \mathrm{eV}$ and $3.77 \mathrm{eV}$ respectively for 216 atoms). These values are in good agreement with previous DFT works $[30,40,41]$. Our main goal is to determine how a NG atom interacts with one of these monovacancies, and if the latter can trap or not the impurity. This could lead to form NG monovacancy complexes (NG-V).

\begin{tabular}{ccc}
\hline $\mathrm{NG}-\mathrm{V}$ & $\mathrm{V}_{S}^{S i}$ & $\mathrm{~V}_{S}^{C}$ \\
\hline $\mathrm{He}$ & $2.00(1.42)$ & $4.13(3.37)$ \\
\hline $\mathrm{Ne}$ & $2.34(2.27)$ & $4.44(3.73)$ \\
\hline $\mathrm{Ar}$ & $4.82(4.33)$ & $6.20(5.51)$ \\
\hline $\mathrm{Kr}$ & $5.70(4.87)$ & $7.12(6.44)$ \\
\hline $\mathrm{Xe}$ & $7.05(6.41)$ & $8.86(8.15)$ \\
\hline
\end{tabular}

Table 3

Formation energies (in eV) of different $\mathrm{NG}$ atoms located in $\mathrm{V}^{S i}$ or $\mathrm{V}^{C}$ using a crystal of 64 atoms. Results calculated using a cell of 216 atoms are given in parentheses.

We will start by defining possible simple NG-V configurations, which are shown in Fig. 1. Firstly, $\mathrm{V}_{S}^{S i}$ or $\mathrm{V}_{S}^{C}$ : these correspond to the case where a NG atom is located at the center of the $\mathrm{V}^{S i}$ or $\mathrm{V}^{C}$ monovacancy, i.e. the $\mathrm{NG}$ atom can be considered in substitution. Secondly, $\mathrm{V}^{S i} \mathrm{~T}$ or $\mathrm{V}^{C} \mathrm{~T}$ : when a NG atom is located in the $\mathrm{T}$ site neighbor of a $\mathrm{Si}$ or $\mathrm{C}$ monovacancy. The crystalline structure of $3 \mathrm{C}-\mathrm{SiC}$ implies that the $\mathrm{T}$ site $1^{\text {st }}$-neighbor of $\mathrm{V}^{S i}$ is necessarily $\mathrm{T}^{S i}$, whereas the $2^{\text {nd }}$-neighbor $\mathrm{T}$ site is $\mathrm{T}^{C}$ (and the opposite for $\mathrm{V}^{C}$ obviously). Thus, $\mathrm{V}^{S i} \mathrm{~T}_{1}^{S i}$ will be used to refer to the initial configuration for a NG atom in the 1st-neighbor $\mathrm{T}_{1}^{S i}$ site, and $\mathrm{V}^{S i} \mathrm{~T}_{2}^{C}$ for a $\mathrm{NG}$ atom initially located in the 2 nd-neighbor $\mathrm{T}_{2}^{C}$ site. The same notation is used to describe the $\mathrm{NG}-\mathrm{V}^{C}$ complexes.

The results of our calculations for $\mathrm{V}_{S}^{S i}$ and $\mathrm{V}_{S}^{C}$ configurations are reported in Table 3. They indicate that both configurations are stable for all $\mathrm{NG}$ atoms, i.e. all $\mathrm{NG}$ atoms are found to remain at the center of $\mathrm{V}^{S i}$ and $\mathrm{V}^{C}$ after structural relaxation. This is in agreement with what was already found by Ginhoven et al [19] for He (0.86 and $1.62 \mathrm{eV}$ for $\mathrm{V}_{S}^{S i}$ and $\mathrm{V}_{S}^{C}$ respectively) using a crystal of 64 atoms. Nevertheless, our computed formation energies appear much larger than theirs, with energy differences of $1.14 \mathrm{eV}$ for $\mathrm{V}_{S}^{S i}$ and $2.51 \mathrm{eV}$ for $\mathrm{V}_{S}^{C}$. Doing the calculations in the larger cell leads to a consequent reduction of the formation energies, but not enough important to close the gap. For the other species, we also found a formation energy reduction when using a large cell, almost constant for $\mathrm{V}^{C}$.

Using now only the smaller cell, we investigated the case of a NG atom located in a $\mathrm{T}$ site $1^{\text {st }}$-neighbor of a monovacancy. Again, He exhibits a specific behavior, different from the other species, and is best described separately. Starting from $\mathrm{V}^{S i} \mathrm{~T}_{1}^{S i}$, the He atom migrates in direction of the monovacancy to finally reach a position located $0.88 \AA$ away from its center. The corresponding formation energy of $1.69 \mathrm{eV}$ is lower than with He in the center of the vacancy. Starting with $\mathrm{He}$ in second neighbor, i.e. in $\mathrm{V}^{S i} \mathrm{~T}_{2}^{C}$, a slightly different final configuration is obtained after relaxation. The formation energy is very close $(1.68 \mathrm{eV})$, as well as the final distance between $\mathrm{He}$ and $\mathrm{V}^{S i}$, but the environments of He in both configurations are 
different. Therefore, around the vacancy, there are several stable locations for He with almost similar energies and short separations, which suggests an easy migration for $\mathrm{He}$ between all of them. Since a lower energy is obtained for He in $\mathrm{V}^{S i} \mathrm{~T}_{1}^{S i}$ and $\mathrm{V}^{S i} \mathrm{~T}_{2}^{C}$, than for He in the center of the vacancy, we have tested the stability of the latter by displacing an He atom initially located in the vacancy by $2 \%$ towards neighboring interstitial sites. Relaxation leads to the migration of He into $\mathrm{V}^{S i} \mathrm{~T}_{1}^{S i}$ or $\mathrm{V}^{S i} \mathrm{~T}_{2}^{C}$ locations. The $\mathrm{V}_{S}^{S i}$ configuration is then weakly stable. Considering now the case of the carbon vacancy, we found that if the $\mathrm{He}$ atom is initially located in $\mathrm{V}^{C} \mathrm{~T}_{1}^{C}$ and $\mathrm{V}^{C} \mathrm{~T}_{2}^{S i}$ configurations, a unique structure is obtained after relaxation. The He-vacancy final distance is $1.27 \AA$, and the corresponding formation energy is $2.45 \mathrm{eV}$. This configuration is also obtained if one starts with $\mathrm{He} 2 \%$ displaced from the center of $\mathrm{V}^{C}$, highlighting the weak stability of He in $\mathrm{V}^{C}$.

The situation is more simple for the other species, with the sole exception of Ne. In fact, we found that a NG atom is stable in both $\mathrm{V}^{C}$ and $\mathrm{V}^{S i}$ configurations, always with a lower formation energy for the latter. This fact has also been pointed by Bertolus [23] for $\mathrm{Kr}$ and Xe. The Fig. 3 represents the variation of formation energies for all stable complexes, as a function of the estimated radius of the NG atom. We obtained a monotonous increase for $\mathrm{V}^{C}$ and $\mathrm{V}^{S i}$ configurations. When the NG atom is located in T sites $1^{\text {st }}$ or $2^{n d}$-neighbor of vacancies, it migrates during relaxation to finally end at the center of $\mathrm{V}^{C}$ or $\mathrm{V}^{S i}$. Only for $\mathrm{Ne}$ and the $\mathrm{V}^{C} \mathrm{~T}_{2}^{S i}$ initial configuration, the migration of Ne during relaxation stopped when the distance between $\mathrm{Ne}$ and the vacancy center is $1.27 \AA$, with a formation energy of $4.01 \mathrm{eV}$. This configuration appears to be very similar to the one obtained in the case of He.

\section{NG divacancy complex}

We also investigated the interaction of $\mathrm{NG}$ atoms with a divacancy $\left(\mathrm{V}^{C} \mathrm{~V}^{S i}\right)$ obtained by removing one $\mathrm{C}$ atom and one $\mathrm{Si}$ atom 1st-neighbor in the zinc-blende lattice. Formation energies of $7.52 \mathrm{eV}$ and $7.54 \mathrm{eV}$ were calculated for $\mathrm{V}^{C} \mathrm{~V}^{S i}$ configuration using 64 and 216 atoms respectively. These results are in fair agreement with values calculated in $[19,42]$. In order to determine the most stable location for a NG atom into the divacancy, different initial configurations were tested. For all species, we considered three possible starting structures: two when the NG atom is located at the ideal position of one vacancy forming the divacancy $\mathrm{V}_{S}^{2}(C)$ or $\mathrm{V}_{S}^{2}(S i)$, and the last one when the NG atom is at the centre between the two vacancies $\mathrm{V}_{C}^{2}$. After structural relaxation, we found a single one final configuration for each species. The NG atom remained in the line passing through the two vacancies forming the divacancy, but with a different position according to the species.

\begin{tabular}{cccc}
\hline $\mathrm{NG}^{-} \mathrm{V}^{2}$ & $E_{f}$ & $\mathrm{~d}\left(\mathrm{NG}-\mathrm{V}^{C}\right)$ & $\mathrm{d}\left(\mathrm{NG}-\mathrm{V}^{S i}\right)$ \\
\hline $\mathrm{He}$ & $1.20(1.19)$ & 1.26 & 0.63 \\
\hline $\mathrm{Ne}$ & $1.85(1.80)$ & 1.29 & 0.60 \\
\hline $\mathrm{Ar}$ & $3.77(3.85)$ & 0.61 & 1.28 \\
\hline $\mathrm{Kr}$ & $4.04(4.15)$ & 0.78 & 1.12 \\
\hline $\mathrm{Xe}$ & $4.36(4.33)$ & 0.95 & 0.95
\end{tabular}

Table 4

Formation energies (in eV) and geometries (in $\AA$ ) of different NG divacancy complexes using a crystal of 64 atoms. Results calculated using a cell of 216 atoms are given in parentheses.

The Table 4 reports all formation energies as well as the distances between the NG atom and the two vacancies, for each species. In the case of $\mathrm{He}$ and $\mathrm{Ne}$, we found that the NG atom is prefentially located closer to $\mathrm{V}^{S i}$, at approximately $1 / 3$ of the initial $\mathrm{V}^{S i}-\mathrm{V}^{C}$ distance. The energy required to insert $\mathrm{He}$ or $\mathrm{Ne}$ into the divacancy is rather small, lower than $2 \mathrm{eV}$. Contrasting results are obtained for $\mathrm{Ar}$ and $\mathrm{Kr}$, since in that case the NG atom is found to be closer to $\mathrm{V}^{C}$, roughly at $1 / 3$ of the $\mathrm{V}^{S i}-\mathrm{V}^{C}$ distance. Formation energies are larger than for $\mathrm{He}$ and $\mathrm{Ne}$, but remains much smaller than interstitial formation energies. Finally, Xe is the only species for which the NG atom is located in the center of the divacancy, the formation energy being $4.3 \mathrm{eV}$ in that case.

\section{Stability analysis}

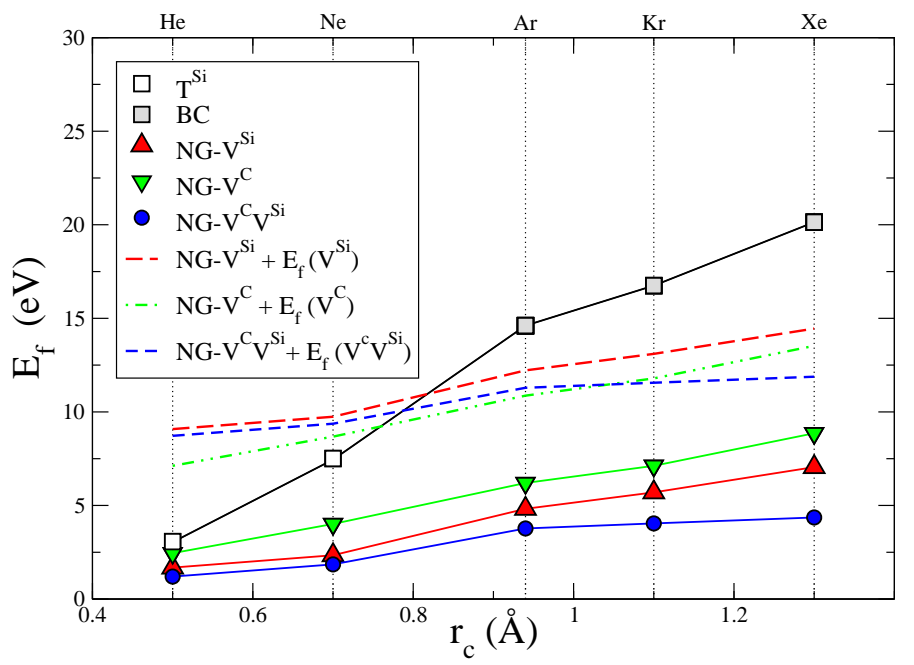

Fig. 4. Formation energies (in eV), caculated using a crystal of 64 atoms, of NG defects as a function of the (estimated) core radius. In dashed line we show the sames curves but adding $\mathrm{E}_{f}^{V^{S i}}, \mathrm{E}_{f}^{V^{C}}$ and $\mathrm{E}_{f}^{V^{C} V^{S i}}$ (color for electronic version).

We have investigated the interaction of NG atoms with the $3 \mathrm{C}-\mathrm{SiC}$ lattice, including or not simple intrinsic point defects such as the mono and divacancy. It is interesting now to compare the formation energies of the different NG 
defects depending on possible conditions. We used here the 64 atoms cell data, but similar results are obtained with 216 atoms data. First we consider a non-damaged silicon carbide crystal, therefore including only a very limited amount of intrinsic defects (vacancies, interstitials) at thermodynamic equilibrium. Then we take into account the energy creation of monovacancies or divacancies for NG complexes. Fig. 4 clearly indicates that in that case, $\mathrm{He}$ and $\mathrm{Ne}$ individual atoms will be located in interstitial positions. However, this is not true anymore for the heaviest NG species like $\mathrm{Ar}, \mathrm{Kr}$, and Xe. In fact, the energy cost for a NG interstitial is so high that the formation of a NG-vacancy complexes becomes favorable, even with the extra cost of vacancies formation. For $\mathrm{Ar}$ and $\mathrm{Kr}$, Fig. 4 suggests that it is energetically cheaper to form a $V^{C}$ monovacancy containing the NG atom. Such a result might be surprising since our calculations have shown that the energy required to put a $\mathrm{NG}$ atom in $V^{C}$ was always higher than in $V^{S i}$, but this point is balanced by the much higher formation energy of $V^{S i}$ compared to $V^{C}$. Finally, for $\mathrm{Xe}$, we found that it is energetically favorable to form a divacancy containing the NG atom. Therefore it is highly unlikely to obtain Ar, $\mathrm{Kr}$ and Xe interstitials in an infinite silicon carbide system at equilibrium.

Considering now the case of a highly damaged silicon carbide, i.e. including a large number of vacancies resulting from implantation for instance, we can compare directly the different formation energies. All NG atoms will prefer to occupy divacancies if available. Then, the second best choice is silicon monovacancies $V^{S i}$. Our results suggest that vacancies are trap for all NG species. This is in qualitative agreement with the previous work from Van Ginhoven et al for He [19]. However, note that in our work, not all possible intrinsic defects have been investigated. For instance, it is known that the silicon monovacancy is metastable, and would evolve to a complex composed of a carbon vacancy and a carbon antisite [43] thanks to thermal activation. It is not clear whether the presence of NG atoms would inhibit this transformation, and makes $V^{S i}$ stable.

\section{Migration}

We investigated the mobility of a single NG atom in a perfect crystal, and located in a tetrahedral site. To compute migration path, as well as the associated migration energy barrier, we performed NEB calculations. We considered He, Ne and Ar species, but not Kr or Xe. The latters are unlikely to exist in tetrahedral configuration as it was shown previously. The same conclusion applies for Ar, but we still consider it to get migration variation trends as a function of NG size.

An initial $\mathrm{T}^{S i}-\mathrm{T}^{C}$ path of 3 intermediate images was

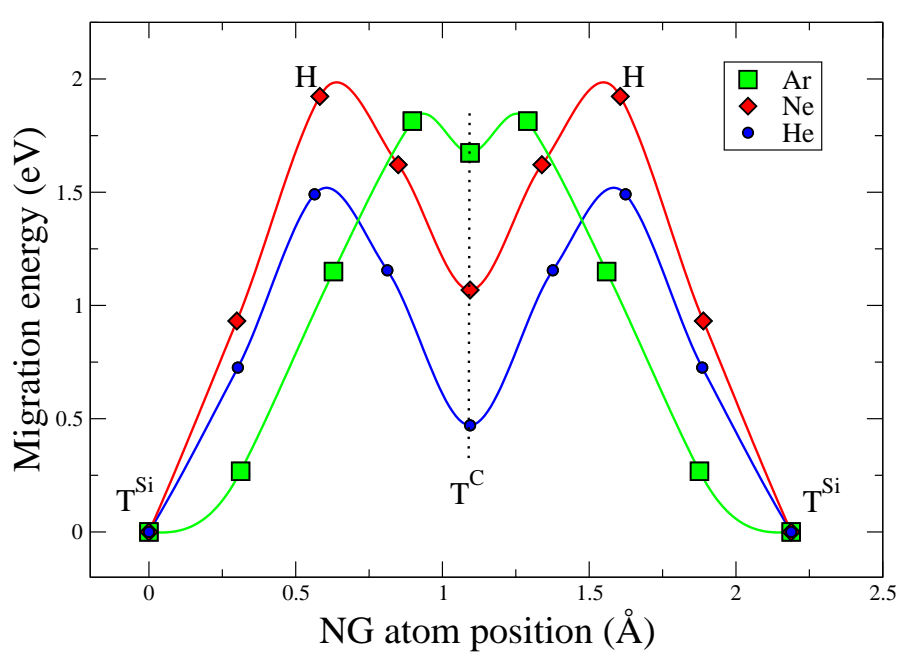

Fig. 5. Calculated energies of $\mathrm{He}, \mathrm{Ne}$ and $\mathrm{Ar}$ atoms, from $\mathrm{T}^{S i}$ configuration to $\mathrm{T}^{S i}$ via $\mathrm{T}^{C}$, as the function of the position of the NG atom along the migration path. Lines are guides for the eyes (color for electronic version).

built for all considered NG atoms, then relaxed to determine the minimum energy migration path between $\mathrm{T}^{S i}$ and $\mathrm{T}^{C}$ sites. After structural relaxation, the $\mathrm{T}^{S i} \mathrm{~T}^{C}$ mechanism was found to be stable. On the Fig. 5, one can remark that the $\mathrm{H}$ configuration is the saddle point for $\mathrm{He}$ and Ne, like in Si [37] or diamond [44]. For Ar the saddle point corresponds to the 4 th image, and is not a high symmetry configuration.

A NG atom located in the $\mathrm{T}^{S i}$ site will diffuse in a perfect $\mathrm{SiC}$ crystal by successive hoppings between, $\mathrm{T}^{S i}$ and $\mathrm{T}^{C}$ sites. The associated calculated migration energy barriers are: 1.49, 1.92 and $1.81 \mathrm{eV}$ for $\mathrm{He}, \mathrm{Ne}$ and Ar respectively. Our calculated value for He is somewhat larger than the experimentally determined activation energy of $1.14 \mathrm{eV}$ measured by Jung [17] for $800 \leq \mathrm{T}(\mathrm{K}) \leq$ 1050. The difference could be explained by a finite temperature effects (lattice thermal expansion, free energy vibrational contributions) not taken into account here. However we obtained a very good agreement with other measurements [16]. Van Ginhoven et al [19] calculated the activation energy of $\mathrm{He}$ interstitial migration to be $2.5 \mathrm{eV}$, for a similar $\mathrm{T}^{S i} \rightarrow \mathrm{T}^{C} \rightarrow \mathrm{T}^{S i}$ path. This much higher value is related to the large energy difference between $\mathrm{T}^{C}$ and $\mathrm{T}^{S i}$ in their work. Although it is difficult to explain such a large discrepancy, a simple argument would suggest that the migration energy is much lower than $2.5 \mathrm{eV}$. In fact, previous studies provide He interstitial migration energy of $0.71 \mathrm{eV}$ in silicon [37] and $2.3 \mathrm{eV}$ in diamond [44]. These values are likely to be bounds for the migration in $\mathrm{SiC}$ since the energy range of defects in this material is typically between those for silicon and diamond. Then, in addition to a better agreement with experiments, our computed value of $1.49 \mathrm{eV}$ seems to be more sound.

For the case of Ne, we found a higher migration energy 
of $1.92 \mathrm{eV}$. Unfortunately, to our knowledge there are no experiments or calculations to compare with.

\section{Conclusion}

Electronic structure calculations were made to study the properties of NG atoms in 3C-SiC. The stability of a NG atom was investigated in the presence or not of a monovancy (or divacancy). In a perfect lattice, the most stable interstitial configuration were $\mathrm{T}^{S i}$ ( $\mathrm{He}$ and $\mathrm{Ne}$ ), and $\mathrm{BC}$ (Ar, $\mathrm{Kr}$ and $\mathrm{Xe}$ ). We found that monovacancy $\mathrm{V}^{S i}$ and $\mathrm{V}^{C}$ are traps for a single NG atom, with a lower formation energy for NG-V $\mathrm{V}^{S i}$. Heavier NG species prefer to occupy the vacancy, whereas He is more stable in its vicinity. NG species tend to remain into divacancies, with low formation energies. A simple analysis demonstrates that at equilibrium, $\mathrm{Ar}, \mathrm{Kr}$ and $\mathrm{Xe}$ interstitials are unlikely to exist since it is energetically cheaper to create a mono or divacancy. This would suggest that the ejection of selfinterstitials should play a major role in the formation and evolution of bubbles filled with heavy NG species. This is in agreement with the experimental observations of a larger density of dislocation loops in the case of Xe compared to $\mathrm{Ne}$ [14]. Finally, we investigated the migration of $\mathrm{He}, \mathrm{Ne}$ and Ar interstitials through successive hoppings between $\mathrm{T}^{S i}$ and $\mathrm{T}^{C}$ sites. The migration energy barriers are determined to be $1.49,1.92$ and $1.81 \mathrm{eV}$ respectively. Using our computed migration and formation energies for He, it can be shown that the energy required for the dissociation of $\mathrm{He}-\mathrm{V}$ or $\mathrm{He}-\mathrm{V}_{2}$ complexes and the subsequent desorption ranges between 3.1 and $3.3 \mathrm{eV}$, in good agreement with experiments $[16,18]$. Note that this could be accidental since we do not consider here the influence of temperature and pressure, which should be taken into account [45]. Our calculations also hinted that the growth of He-filled bubbles is likely to happen through the migration of He interstitials. Nevertheless, it will be necessary to investigate the migration of NG-mono or divacancy complexes, for He but also for larger NG atoms, to draw more definitive conclusions.

\section{Acknowledgments}

Marjorie Bertolus is gratefully acknowledged for fruitful discussions. This work was partly supported by the GNR MATINEX.

\section{References}

[1] W. J. Choyke, G. Pensl, Physical properties of sic, Mater. Res. Soc. Bull. 22 (3) (1997) 25.

[2] S. E. Donnelly, J. H. Evans (Eds.), Fundamental Aspects of Inert Gases in Solids, Plenum, 1991.

[3] P. R. Heck, K. K. Marhas, P. Hoppe, R. Gallino, H. Baur, R. Wieler, Presolar he and ne isotopes in single circumstellar sic grains, The Astrophysical Journal 656 (2007) 1208.
[4] S. E. Donnelly, The density and pressure of helium in bubbles in implanted metals: a critical review, Radiation Effects 90 (1985) 1.

[5] G. F. Cerofolini, F. Corni, S. Frabboni, C. Nobili, G. Ottaviani, R. Tonini, Hydrogen and helium bubbles in silicon, Mater. Sci. Eng. Reports 27 (2000) 1.

[6] E. Oliviero, S. Peripolli, L. Amaral, P. F. P. Fichtner, M. F. Beaufort, J. F. Barbot, S. Donnelly, Damage accumulation in neon implanted silicon, J. Appl. Phys. 100 (2006) 043505.

[7] M.-L. David, F. Pailloux, V. Mauchamp, L. Pizzagalli, In situ probing of helium desorption from individual nanobubbles under electron irradiation, Appl. Phys. Lett. 98 (2011) 171903.

[8] K. Hojou, K. Izui, Bubbles in sic crystalas formed by helium ion irradiation at high temperatures, J. Nucl. Mater. 160 (1988) 147.

[9] K. Hojou, S. Furuno, K. Kushita, H. Otsu, Y. Furuya, K. Izui, In situ eels and tem observation of silicon carbide irradiated with helium ions at low temperature and successively annealed, Nucl. Instrum. Methods Phys. Res. B 116 (1996) 382.

[10] J. Chen, P. Jung, H. Trinkaus, Evolution of helium platelets and associated dislocation loops in $\alpha$-sic, Phys. Rev. Lett. 82 (13) (1999) 2709.

[11] J. Chen, P. Jung, H. Trinkaus, Microstructural evolution of helium-implanted $\alpha$-sic, Phys. Rev. B 61 (19) (2000) 12923.

[12] P. Jung, H. Klein, J. Chen, A comparison of defects in helium implanted $\alpha$ - and $\beta$-sic, J. Nucl. Mater. 283-287 (2000) 806.

[13] L. Vincent, T. Sauvage, G. Carlot, P. Garcia, G. Martin, M.-F. Barthe, P. Desgardin, Thermal behaviour of helium in silicon carbide: influence of microstructure, Vacuum 83 (2009) S36.

[14] C. Zhang, Y. Sun, Y. Song, T. Shibayama, Y. Jin, L. Zhou, Defect production in silicon carbide irradiated with ne and xe ions with energy of $2.3 \mathrm{mev} / \mathrm{amu}$, Nucl. Instrum. Methods Phys. Res. B 256 (2007) 243.

[15] W. R. Allen, The lattice location of helium implanted in $\alpha$-sic, J. Nucl. Mater. 210 (1994) 318.

[16] E. Oliviero, A. van Veen, A. Fedorov, M.-F. Beaufort, J.-F. Barbot, Helium implantation defects in sic studied by thermal helium desorption spectrometry, Nucl. Instrum. Methods Phys. Res. B 186 (2002) 223.

[17] P. Jung, Diffusion and retention of helium in graphite and silicon carbide, J. Nucl. Mater. 191-194 (1992) 377.

[18] S. Miro, J. Constantini, J. Haussy, L. Beck, S. Vaubaillon, S. Pellegrino, C. Meis, J. Grob, Y. Zhang, W. Weber, Nuclear reaction analysis of helium migration in silicon carbide, J. Nucl. Mater. 415 (2011) 5.

[19] R. M. V. Ginhoven, A. Chartier, C. Meis, W. J. Weber, L. R. Corrales, Theoretical study of helium insertion and diffusion in 3c-sic, J. Nucl. Mater. 348 (2006) 51.

[20] J. H. Kim, Y. D. Kwon, P. Yonathan, I. Hidayat, J. G. Lee, J.-H. Choi, S.-C. Lee, The energetics of helium and hydrogen atoms in $\beta$-sic: an ab initio approach, J. Mater. Sci. 44 (2009) 1828.

[21] A. Couet, J.-P. Crocombette, A. Chartier, Atomistic study of the thermodynamic equilibrium of nano-sized helium cavities in Bsic, J. Nucl. Mater. 404 (2010) 50.

[22] W. Cheng, M.-J. Ying, F.-S. Zhang, H.-Y. Zhou, S.-F. Ren, Density functional study on helium and hydrogen interstitials in silicon carbide, Nucl. Instrum. Methods Phys. Res. B 269 (2011) 2067.

[23] M. Bertolus, HDR Thesis, Universit de la Méditerranée, Report CEA-R-6281, ISSN 0429-3460 (2011) ; http://tel.archivesouvertes.fr/tel-0066525.

[24] P. Hohenberg, W. Kohn, Inhomogeneous electron gas, Phys. Rev. 136 (3B) (1964) B864.

[25] W. Kohn, L. J. Sham, Self-consistent equations including exchange and correlation effects, Phys. Rev. 140 (4A) (1965) A1133.

[26] P. Giannozzi, S. Baroni, N. Bonini, M. Calandra, R. Car, C. Cavazzoni, D. Ceresoli, G. L. Chiarotti, M. Cococcioni, I. Dabo, A. Dal Corso, S. de Gironcoli, S. Fabris, 
G. Fratesi, R. Gebauer, U. Gerstmann, C. Gougoussis,

A. Kokalj, M. Lazzeri, L. Martin-Samos, N. Marzari, F. Mauri,

R. Mazzarello, S. Paolini, A. Pasquarello, L. Paulatto,

C. Sbraccia, S. Scandolo, G. Sclauzero, A. P. Seitsonen,

A. Smogunov, P. Umari, R. M. Wentzcovitch, Quantum espresso:

a modular and open-source software project for quantum simulations of materials, J. Phys.: Condens. Matter 21 (39) (2009) 395502 (19pp).

URL http://www. quantum-espresso.org

[27] D. Vanderbilt, Soft self-consistent pseudopotentials in a generalized eigenvalue formalism, Phys. Rev. B 41 (11) (1990) 7892.

[28] H. J. Monkhorst, J. D. Pack, Special points for brillouin-zone integrations, Phys. Rev. B 13 (12) (1976) 5188.

[29] J. P. Perdew, K. Burke, M. Ernzerhof, Generalized gradient approximation made simple, Phys. Rev. Lett. 77 (18) (1996) 3865 .

[30] G. Lucas, Ph. D, Université de Poitiers (2006).

[31] J. M. Lento, L. Torpo, T. E. M. Staab, R. M. Nieminen, Selfinterstitials in 3c-sic, J. Phys.: Condens. Matter 16 (2004) 1053.

[32] G. Lucas, L. Pizzagalli, Structure and stability of irradiationinduced frenkel pairs in 3c-sic using first principles calculations, Nucl. Instrum. Methods Phys. Res. B 25 (2007) 124.

[33] T. Liao, G. Roma, J. Wang, First-principles study of neutral silicon interstitials in 3c- and 4h-sic, Philos. Mag. 89 (26) (2009) 2271 .

[34] A. Gali, P. Deak, E. Rauls, N. T. Son, I. G. Ivanov, F. H. C. Carlsson, E. Janzen, W. J. Choyke, Phys. Rev. B 67 (2003) 155203.

[35] H. Jónsson, G. Mills, K. W. Jacobsen, Nudged elastic band method for finding minimum energy paths of transitions, in: B. J. Berne, G. Ciccotti, D. F. Coker (Eds.), Classical and Quantum Dynamics in Condensed Phase Simulations, World Scientific, 1998, Ch. 16, p. 385.

[36] G. Henkelman, H. Jónsson, Improved tangent estimate in the nudged elastic band method for finding minimum energy paths and saddle points, J. Chem. Phys. 113 (22) (2000) 9978.

[37] A. Charaf Eddin, G. Lucas, M. F. Beaufort, L. Pizzagalli, Dft calculation of the stability and mobility of noble gas atoms in silicon, Comput. Mat. Sci. 44 (2009) 1030.

[38] M. Alatalo, M. J. Puska, R. M. Nieminem, First-principles study of he in si, Phys. Rev. B 46 (19) (1992) 12806.

[39] S. K. Estreicher, J. Weber, A. Derecskei-Kovacs, D. S. Marynick, Noble-gas-related defects in si and the origin of the $1018 \mathrm{mev}$ photoluminescence line, Phys. Rev. B 55 (8) (1997) 5037.

[40] A. Zywietz, J. Furthmuller, F. Bechstedt, Phys. Rev. B 59 (1999) $15166-15180$

[41] M. Salvador, J. M. Perlado, A. Mattoni, F. Bernardini, L. Colombo, Defect energetics of $\beta$-sic using a new tight-binding molecular dynamics model, J. Nucl. Mater. 329-333 (2004) 1219.

[42] L. Torpo, T. E. M. Staab, R. M. Nieminen, Phys. Rev. B 65 (2002) 085202

[43] M. Bockstedte, A. Mattausch, O. Pankratov, Ab initio study of the migration of intrinsic defects in 3c-sic, Phys. Rev. B 68 (2003) 205201.

[44] J. Goss, R. Eyre, P. Briddon, A. Mainwood, Density functional simulations of noble-gas impurities in diamond, Phys. Rev. B 80 (2009) 085204.

[45] A. Charaf Eddin, L. Pizzagalli, First-principles calculations of helium and neon desorption from cavities in silicon, J. Phys.: Condens. Matter 24 (2012) 175006 